\title{
Resin infiltration technique as minimal invasive approach for treatment of mild to moderate dental fluorosis in an adolescent: a case report
}

Muhammad Nur Izham Khairuddin, Pengiran Muhammad Badi'uzzaman Awang Iskanderdzulkarnein, Mohd Haikal Mohd Halil*

Department of Restorative Dentistry, Kulliyyah of Dentistry, International Islamic University Malaysia, Kuantan Campus, 25250 Kuantan, Pahang, Malaysia

\begin{abstract}
Dental fluorosis can be defined as a developmental condition that affects dental hard tissue, mainly enamel characterised with white or yellowish lesions due to excessive fluoride exposure. Fluorosis can have a major impact on the appearance, structure and shape of the tooth which posed a significant aesthetic concern to individuals having this condition. There are several treatments recommended in treating dental fluorosis depending on the severity of the disease itself ranging from tooth bleaching to prosthetic crowns in severe cases. This case report describes the use of resin infiltration technique on a patient with mild to moderate severity of dental fluorosis of the upper anterior teeth which produce an acceptable improvement of the appearance of the affected tooth. Resin infiltration technique in this case provided a conservative and inexpensive approach in treating mild to moderate dental fluorosis for the patient, improving the aesthetic without significant loss of tooth structure.
\end{abstract}

Keywords: dental fluorosis, resin infiltration, aesthetic, minimally invasive technique

\section{Introduction}

Dental fluorosis can be defined as a developmental condition of enamel caused by excessive absorption and repeated exposure to low doses of fluoride during all stages of tooth development (Pamela Denbesten \& Li, 2011). This causes disruption in enamel development and results in hypomineralization of the enamel and giving the appearance of white or yellowish lesions on tooth surfaces (Aoba \&
Received:

20 June 2021

Revised:

19 July 2021

Accepted:

27 July 2021

Published Online:

31 July 2021

How to cite this article:

Khairuddin, M. N. I., Awang Iskanderdzulkarnein, P. M. B. ., \& Mohd Halil, M. H. . (2021). Resin Infiltration Technique as Minimal Invasive Approach Towards Mild to Moderate Dental Fluorosis in an Adolescent: a Case Report. IIUM Journal of Orofacial and Health Sciences, 63-72. Retrieved from https://doi.org/10.31436/ijohs. v2i2.98

\section{Article DOI:}

https://doi.org/10.31436/ijohs. v2i2.98

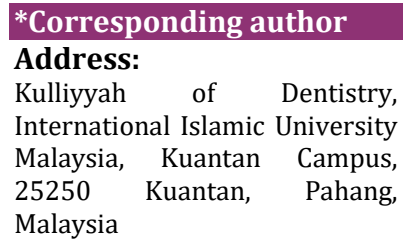

Telephone: +60192816007 Email address:

drhaikal@iium.edu.my
Fejerskov, 2002). Fluoride disturbs enamel formation by reducing calcium ion concentration in the matrix which then interferes with protease activity, thus delaying or inhibiting enamel matrix protein degradation (Fejerskov et al., 1990; Robinson et al., 2004). The main sources of fluoride that are likely to cause dental fluorosis usually come from the caries prevention modalities such as accidental ingestion of fluoride-containing dental 
products (e.g. toothpaste, varnishes, gels, fluoride supplement) and greater than 1 ppm of added or naturally occurring fluoride in drinking water (Watts \& Addy, 2001). Dental fluorosis cases had been reported worldwide with Saudi Arabia reported a high overall prevalence of $46.52 \%, 11.3 \%$ reported from Germany and as low as $4 \%$ from India (Alshammary et al., 2020). Dental fluorosis also can be seen quite regularly in Malaysia as a study show prevalence of slightly above the optimal levels of exposure at $57.8 \%$, particularly for mild to moderate cases in fluoridated areas of Malaysia (Tan et al., 2005). A recent study has shown a lower prevalence of fluorosis amongst children who lived in a place where water fluoridation has been stopped and were exposed partially to fluoride through toothpaste or foods (Karim et al., 2021).

Clinically, dental fluorosis can be seen as diffuse, symmetrical, discoloured white opaque stains and striations in mild to moderate cases. However, in severe forms; porosity, pitting, and brownish areas associated with fragile enamel can often be seen on the surface of the tooth. Nevertheless, an accurate diagnosis of the disease should only be reached from proper anamnesis, as not to be confused with other types of tooth discolouration and staining that shows quite similar clinical presentation such as the hypomaturation type of Amelogenesis Imperfecta (Crawford \& Aldred, 1992). The amount and duration of fluoride exposure in addition to the stages of enamel development during exposure play a role in determining the severity of the fluorosis which require different treatment measures (Bertassoni et al., 2008). It also has been suggested that the direct effect of fluoride on ameloblast may have influences on the development of fluorosed enamel. It includes structural changes of early secretory ameloblasts, cell proliferation alteration, reduced protein synthesis, apoptosis, the elevation of F-actin and stress-related protein upregulation (Pamela Denbesten \& Li, 2011). Fluoride also affects the cell-matrix interactions during tooth formation which also have a role in the development of fluorosis by hindering hydrolysis of amelogenin which causes a delay in final enamel matrix mineralization (Debensten et al., 1985).

Based on Dean's Fluorosis Index (DFI), fluorosis can be categorized by scoring according to the clinical presentation of the lesion itself as in Table 1 (Pamela Denbesten \& Li, 2011; Tirlet et al., 2013). Several treatment options are available for the treatment of dental fluorosis depending on the severity and extent of the disease. For mild to moderate levels of fluorosis, dental bleaching, micro abrasion or a combination of both has been recommended and shown considerable success (Sherwood, 2010). Veneers also have been shown to result in successful outcomes in the management of moderate levels of fluorosis (Auschill et al., 2015). A more invasive technique is needed in managing severe fluorosis with the prescription of prosthetic crowns, especially when there is a mottling appearance and loss of occlusal vertical dimension of the patient. This is due to the common findings of severely fluorosed enamel that easily fractured even during normal functional use (McKay, 1952).

A technique derived from the enamel infiltration procedure developed for incipient caries management has been applied in dental fluorosis treatment as well (Wang et al., 2020). The resin infiltration technique which involves three-step of etching, drying and infiltrating resin into the porous enamel has shown promising outcomes in the management of mild to moderate levels of fluorosis. This procedure is more conservative in nature and conforms to the concept of minimally invasive dentistry. This technique masks the appearance of white lesions by its additional positive effect due to the similar refractive index of the resin and enamel itself (Giambro et al., 1995). It has also demonstrated good clinical applicability amongst the clinicians and high acceptance by patients as reported by Kugel and colleague (Kugel et al., 2009).

This case report will provide a step-by-step procedure of resin infiltration technique for a moderate level of fluorosis and the aesthetic outcome. 
Table 1. Fluorosis index of H.T. Dean (1942)

\begin{tabular}{|ll|}
\hline \multicolumn{1}{|c|}{ Score } & \multicolumn{1}{c|}{ Criteria } \\
Normal (0) & Normal teeth with a smooth, uniform natural coloured tooth surface \\
Very mild (1) & $\begin{array}{l}\text { Teeth that have some white flecks or spots } \\
\text { No more than } 25 \% \text { of the tooth is covered with small white opaque } \\
\text { areas }\end{array}$ \\
Mild (2) & $\begin{array}{l}\text { Less than } 50 \% \text { of the tooth affected with white opaque areas } \\
\text { Moderate (3) }\end{array}$ \\
associated with brown staining \\
Teeth that are severely mottled or pitted and often have brown \\
staining which affects $100 \%$ of the enamel surface of the tooth
\end{tabular}

\section{Case report}

A 22-year-old male student was referred to the Department of Restorative Dentistry Specialist Clinic, International Islamic University of Malaysia (IIUM) seeking treatment to improve the aesthetic appearance of his upper front teeth. His main complaint is that his teeth exhibited white discolouration which he started to notice in high school along with the abnormal shape of the lateral incisors, and affecting his selfesteem whenever he smiles. Upon intra-oral examination and anamnesis, there was generalised fluorosis was observed, with mild to moderate severity with the score of 2 and 3 based on Dean's Fluorosis Index (DFI) on both upper and lower anterior teeth (Figure 1). It was also noted that upper and lower posterior teeth have severe fluorosis with a score of 4 from DFI. The upper front teeth also had mild spacing and peg-shaped of right and left lateral incisors (Figure 2). The patient demonstrated optimal oral hygiene level with satisfactory periodontal health and is systemically healthy. The initial treatment plan proposed and agreed to by the patient, which was to mask the mild to moderate fluorosis of upper anterior teeth with resin infiltration and followed by the placement of direct composite veneer on the peg-shaped lateral incisors. However, the patient decided to defer the composite veneer treatment due to financial constraints.

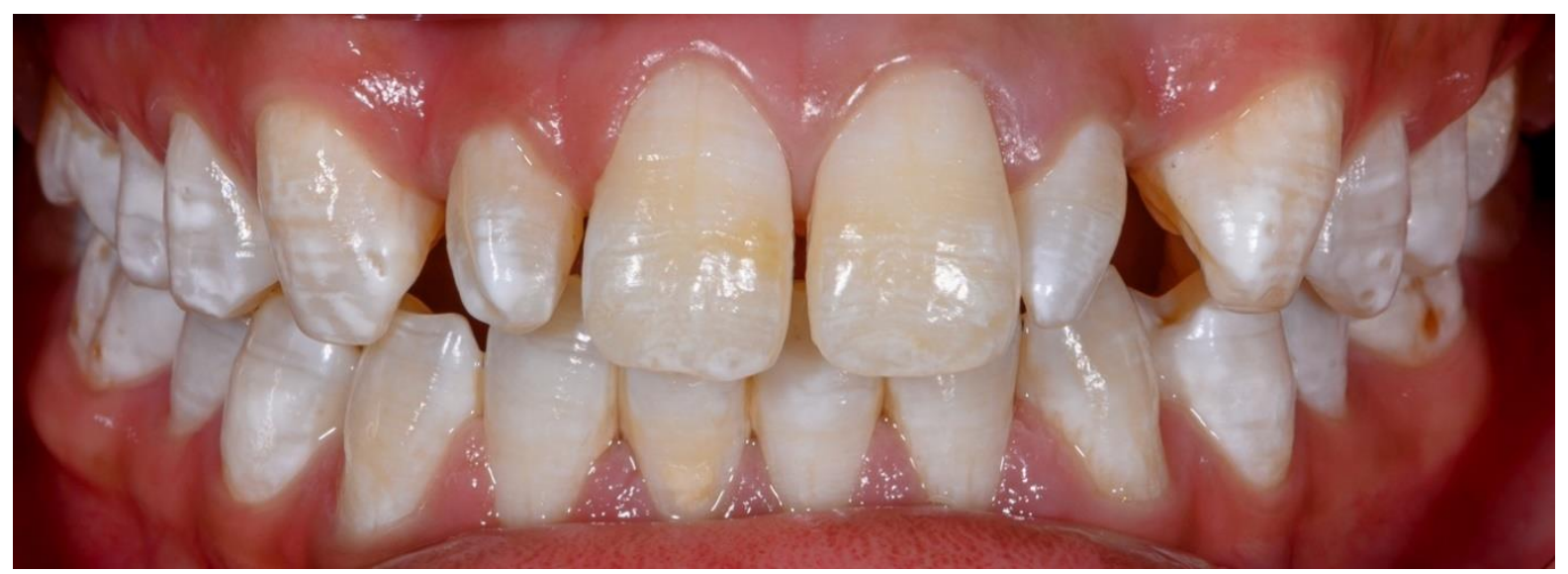

Figure 1. Intra-oral view of the patient when during the initial presentation to the clinic. 


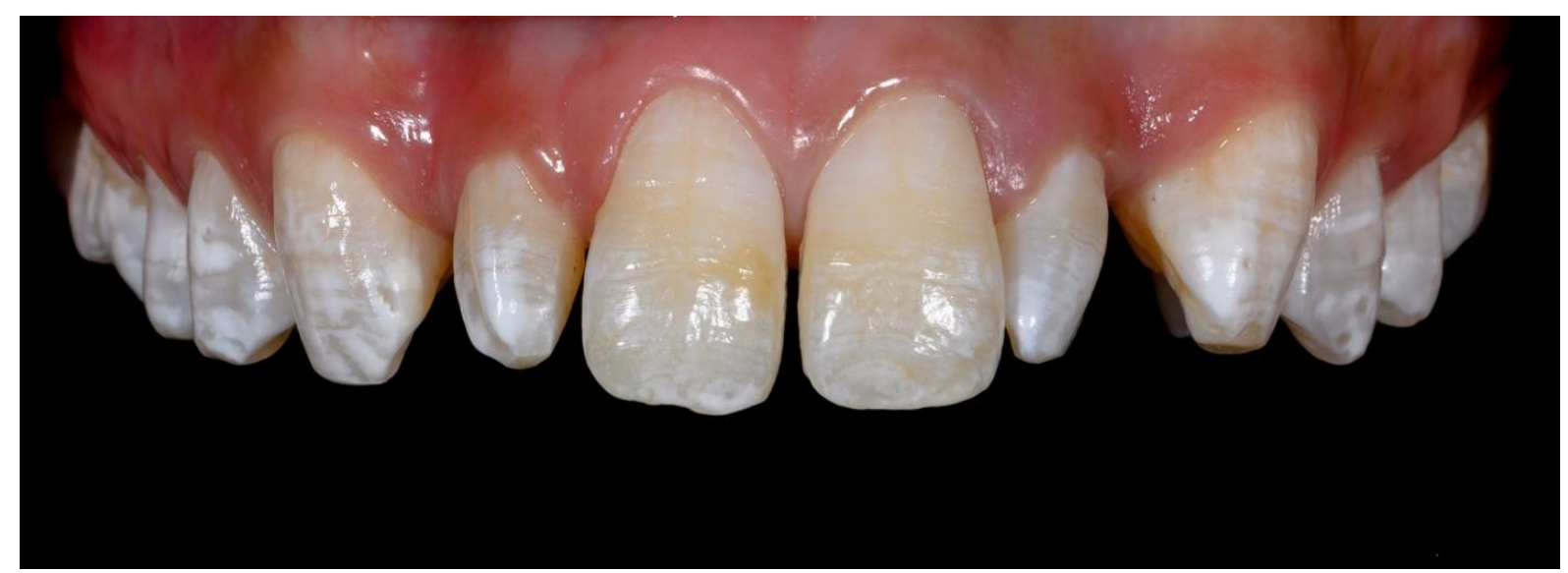

Figure 2. Mild to moderate fluorosis of upper anterior teeth span from tooth 13 to 23 . Notice the peg-shaped of both the right and left lateral incisors (Tooth 12 and 22).

Prior to the commencement of the resin infiltration procedure, scaling and dental prophylaxis was carried to ensure the teeth are clean, free of plaque and calculus. Excess prophylaxis paste was then rinsed away using a water syringe. Rubber dam was placed using multiple isolations from the upper right first molar to the contralateral left molar. Floss ligatures were tied to the respective teeth to secure rubber dam inversion, therefore protecting the soft tissue and ensure a clean and dry working area (Figure 3).

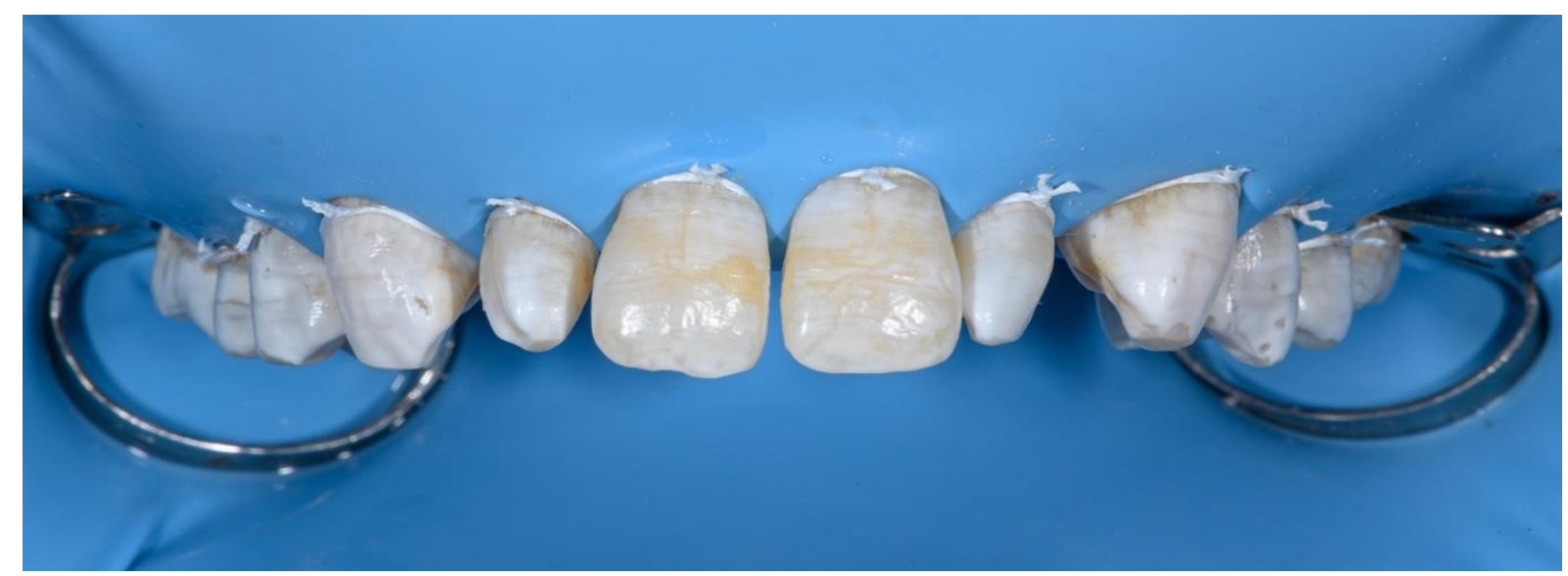

Figure 3. The whitish spot of the fluorosis become accentuated due to lack of moisture from the rubber dam isolation

Following the manufacturer's instructions, resin infiltration procedure (Icon, DMG Chemisch-Pharmazeutische Fabrik $\mathrm{GmbH}$, Hamburg, Germany) was initiated on the maxillary incisors (DFI $=2$ ), canines (DFI = 2 ) and premolars (DFI $=3$ ). Premolars were included in the procedure to assess the effectiveness of resin infiltration on a severe level of fluorosis lesion.

The labial enamel surface of the teeth was etched with $15 \%$ hydrochloric acid gel (ICON-Etch, DMG Chemisch-
Pharmazeutische Fabrik GmbH, Hamburg, Germany) for 120 seconds (Figure 4). The etchant gel was then rinsed away for 30 seconds using water spray and gently dried. At this stage, frosty appearance will become more evident on the fluorosed area. The etching step was then repeated another two times before the application of drying agent (ICON-Dry, DMG ChemischPharmazeutische Fabrik GmbH, Hamburg Germany) applied onto the tooth surface for 30 seconds to ensure desiccation of the enamel surface (Figure 5). 


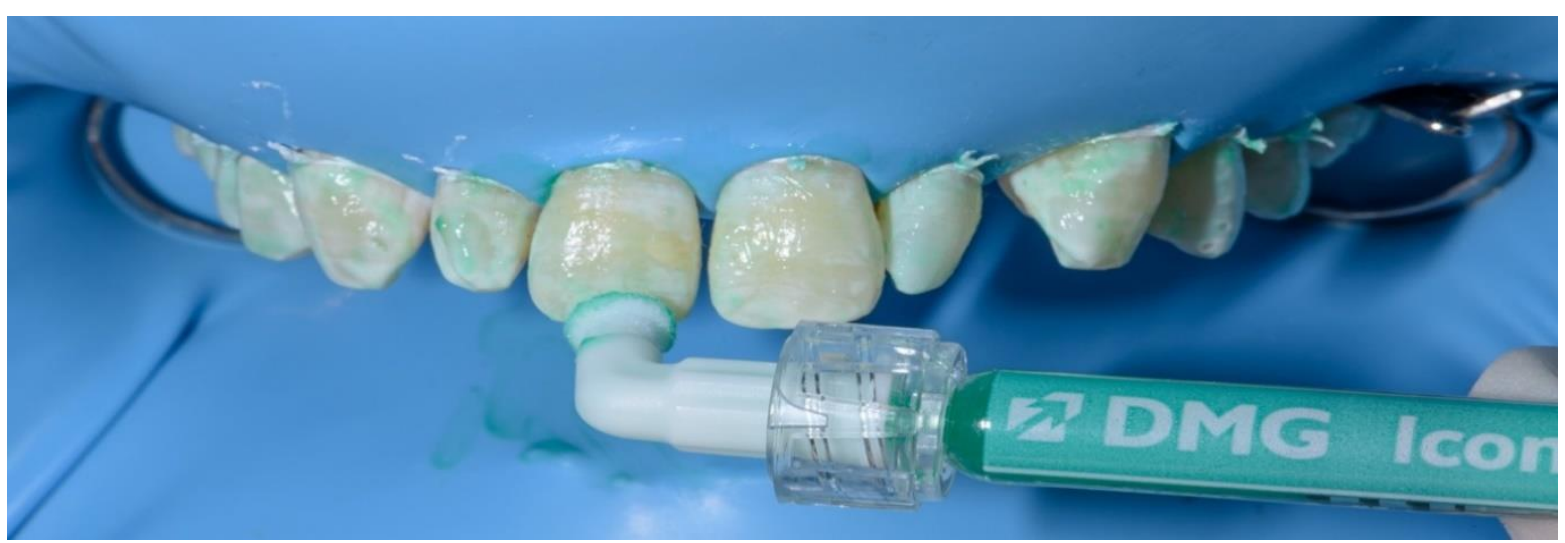

Figure 4. The application of etching gel on the tooth surface.

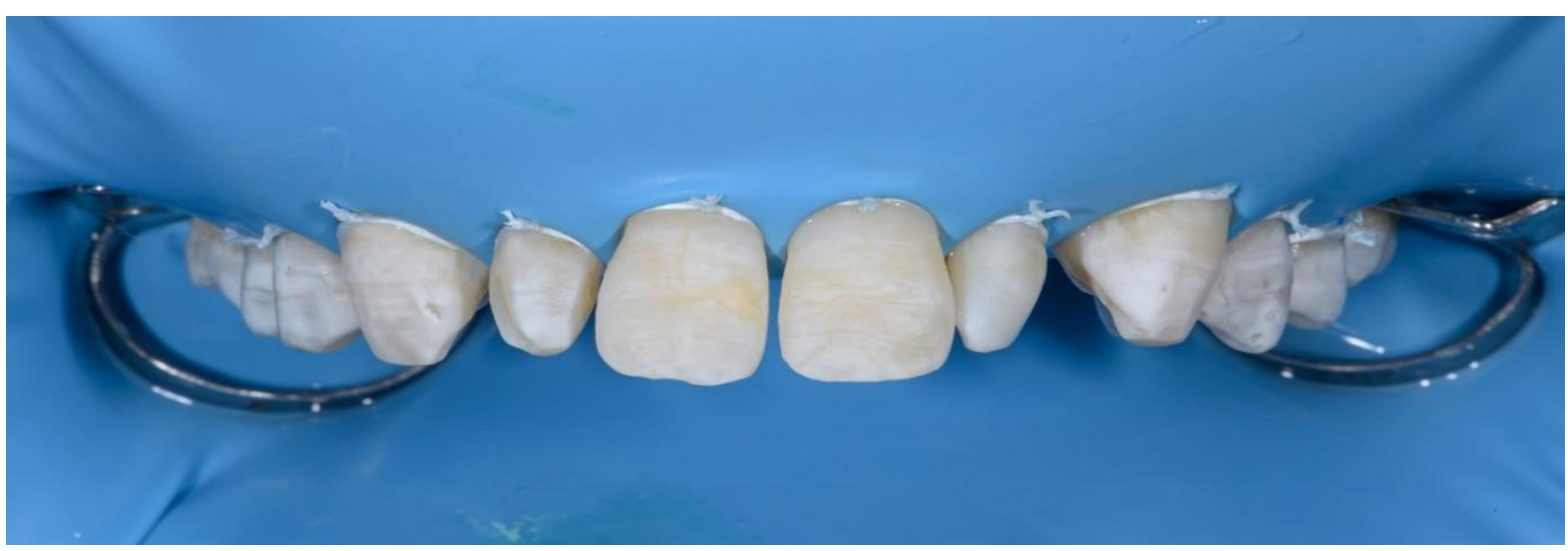

Figure 5. Tooth surfaces were completely dried using the drying agent, ICON-Infiltrant. Notice the premolars still show marked opacities even after the repeated etching cycle done on the teeth.

Before resin infiltrant was applied, the affected enamel surface was assessed, on whether the opacities decreased or disappeared, indicating the treatment effectiveness. For this patient, the authors decided to repeat the etching cycle on premolars to see the effect of the additional etching cycle showed an improvement of the severe opacities which however were still quite visible afterwards. The drying agent also serves as a preview of the aesthetic outcome that can be expected after the infiltration. Subsequently, resin infiltrant agent containing tetramethylene glycol dimethacrylate (TEGDMA) (ICON-Infiltrant, DMG Chemisch-Pharmazeutische Fabrik $\mathrm{GmbH}$, Hamburg, Germany) was applied on the treated surface and allowed to penetrate for three minutes. This was deemed adequate in ensuring the maximal infiltration process (Figure 6). Excess resin infiltrant was removed using a cotton roll and dental floss for interproximal areas, followed by light-curing on each tooth for 40 seconds.

The rubber dam was then removed and the surface was polished using flexible diamondimpregnated spirals (EVE Diacomp Plus Twist, EVE Ernst Vetter GmbH; Pforzheim, Germany) to ensure a smooth and aesthetically acceptable glossy surface finish (Figures 7, 8, 9, and 10). The patient was satisfied with the final outcome of the treatment. There was a marked improvement in the appearance of the concerned tooth as white opacities managed to be masked following the treatment. The final shade of the teeth also in the authors' opinion will be much simpler to be followed in the subsequent direct composite veneer procedure as it eliminates the need to use white stain to mimic the fluorosis. 


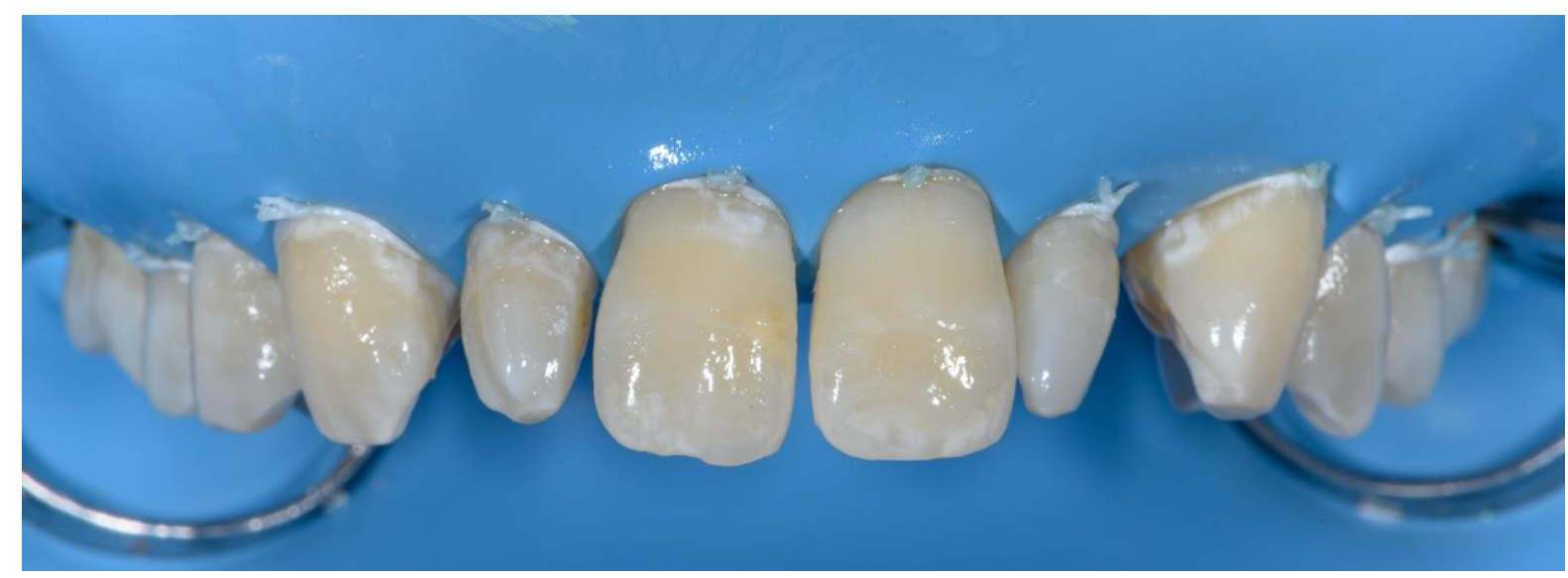

Figure 6. Immediate view after the application of resin infiltrant.

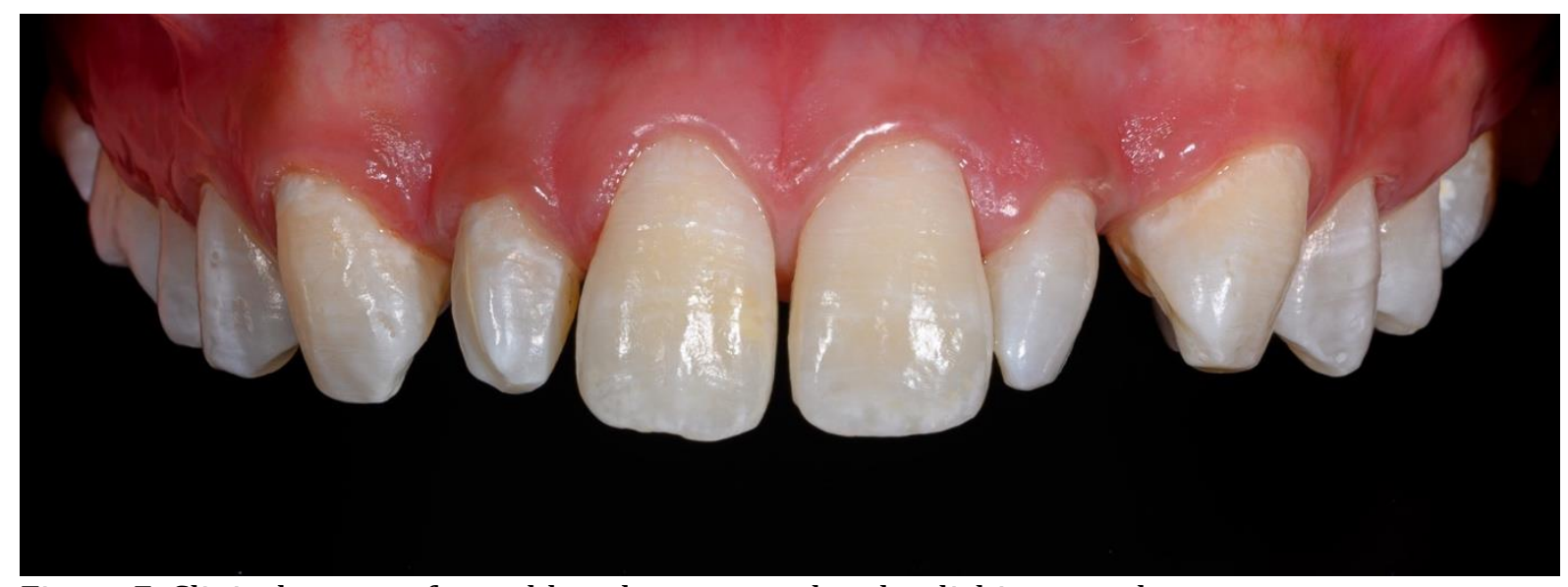

Figure 7. Clinical aspect after rubber dam removal and polishing was done.

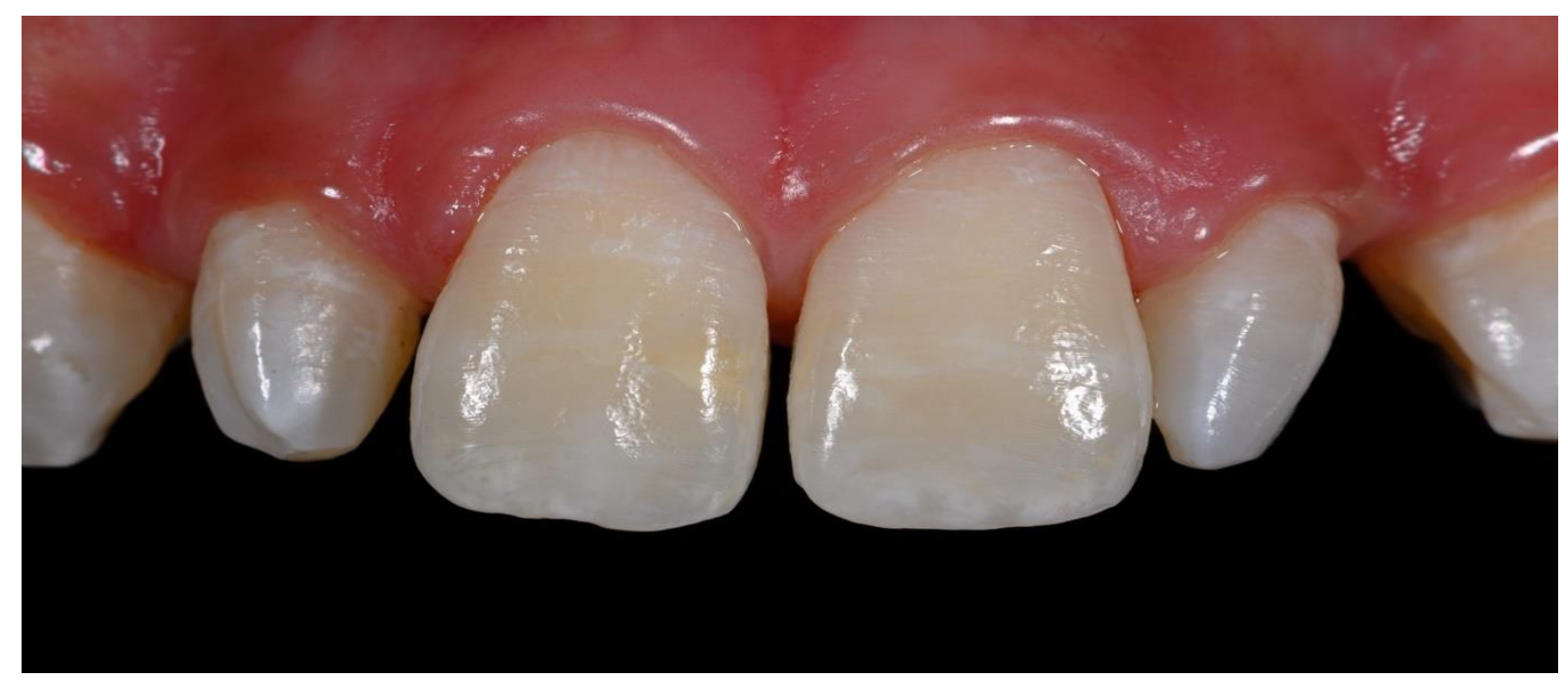

Figure 8. A close-up view of the resin infiltrated maxillary incisors. 


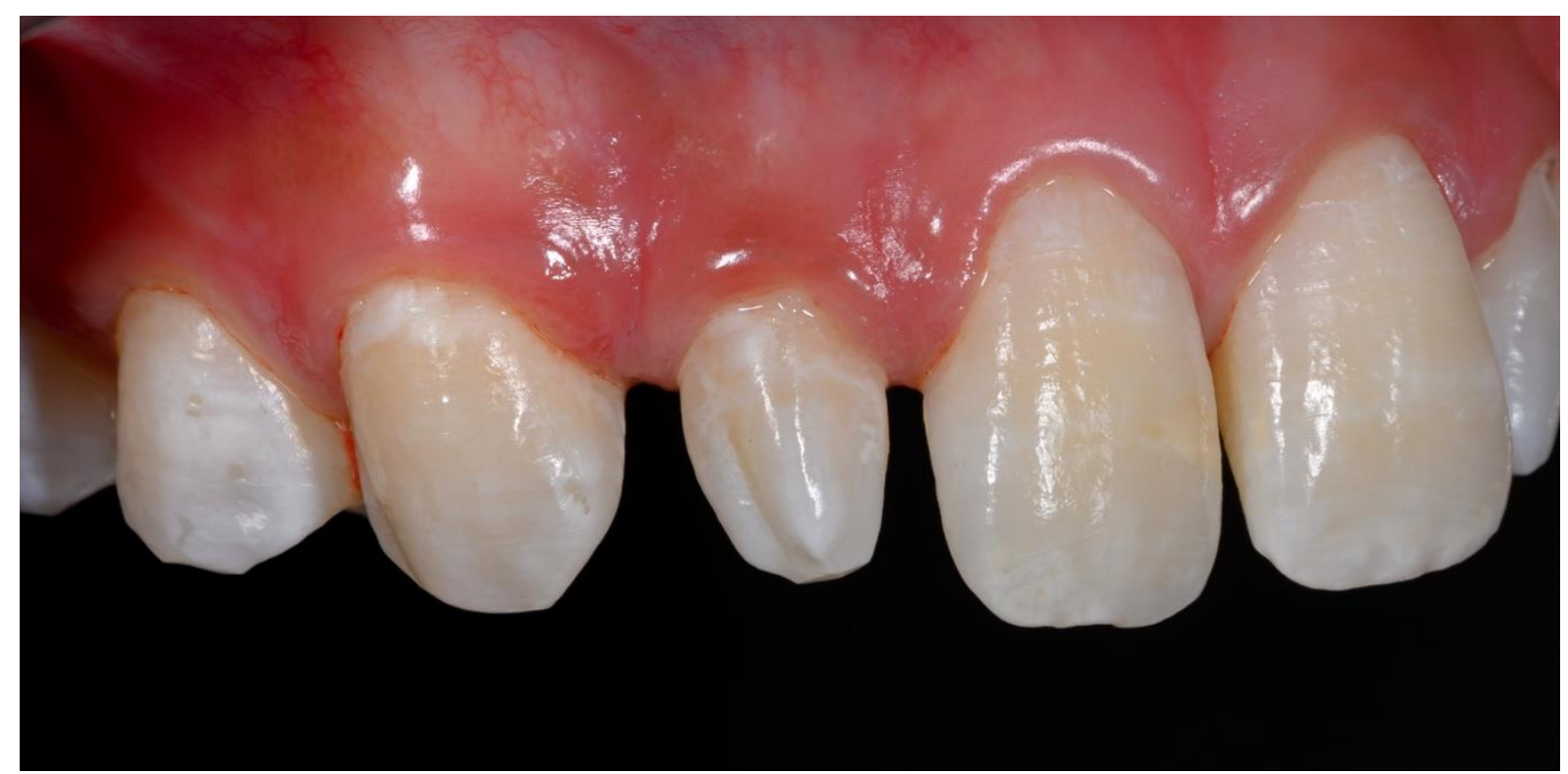

Figure 9. A close-up view of the right side, showing the resin-infiltrated teeth.

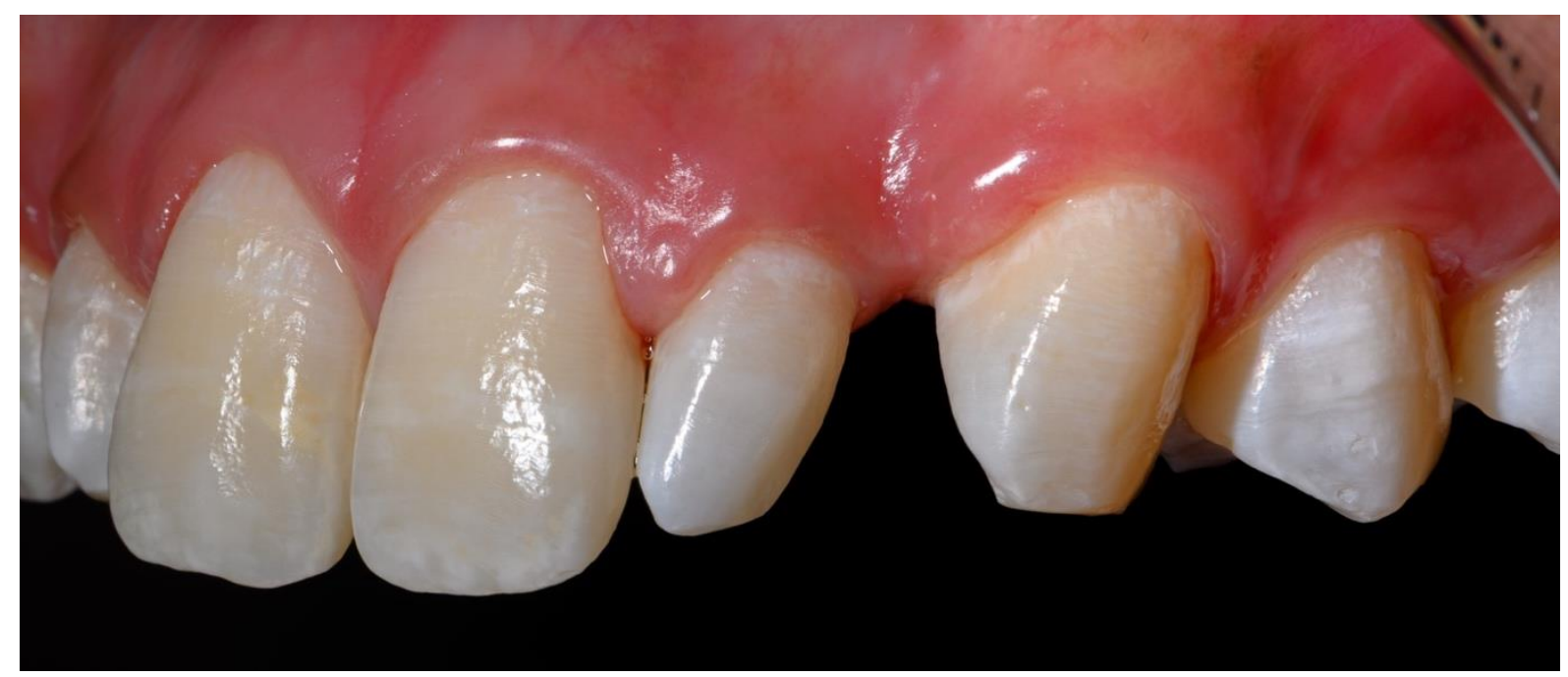

Figure 10. A close-up view of the left side, showing the resin infiltrated teeth.

\section{Discussion}

Tooth discolouration, especially dental fluorosis poses an aesthetic concern amongst many individuals especially in the anterior region where it is more visible to other people. It also can affect both selfconfidence and people's perception of aesthetics, especially in younger individuals. This may have a profound impact on their psychosocial life in severe cases of dental fluorosis (Narayanan et al., 2019; DuarteRodrigues et al., 2020). It is also noted that nowadays patients are more inclined towards a non-invasive approach in having procedures done to their teeth (Frencken et al., 2012). For the patient described in this case report, the resin infiltration technique was successful in giving aesthetic improvement of the appearance of the concerned teeth with a non-invasive approach.

For this patient, it was noted that the remaining part of the dentition, especially posterior to the teeth of concern was presented with severe fluorosis. The attempt to reduce the white discolouration and pitting of the posterior tooth was partially successful. As the whitish appearance is 
somewhat reduced, the appearance is still considered suboptimal in aesthetic value. Therefore, in the authors' opinion, case selection is very important in determining the success of the resin infiltration technique. Patients with a severe level of fluorosis should be given the option of a combination approach, or more invasive restorative treatment such as cosmetic veneer. However, as the primary concern of the patient in this case only involved the appearance of his upper anterior teeth, such options were excluded. As the patient also required subsequent restorative treatment to close midline diastema and direct composite veneer on peg lateral incisors, application of an adhesive system during the direct resin composite procedure will provide higher bond strength as suggested by Wiegand and colleagues (Wiegand et al., 2011). In the authors' opinion, shade matching will be much easier if the technique was done before the restorative procedure commencement as it will simplify the layering technique in the direct composite restoration.

Resin infiltration technique was also chosen because the patient is still young and any attempt to provide prosthetic treatment will result in unnecessary removal of tooth structure from relatively virgin teeth. Other treatments options were excluded in this patient as this approach is more costeffective and can produce an immediate positive result in one appointment when compared with direct and indirect veneer. In addition, the procedure is pain-free as it eliminates the need to use local infiltration and as be seen in other restorative procedures (Auschill et al., 2015). Microabrasion is not suitable for this case as it has only been shown effective in treating milder cases of fluorosis and concern with the removal of tooth structure (Shenoi et al., 2012.). There is a lack of consensus regarding the duration and the repetition cycles needed in the micro-abrasion technique to achieve the optimal result without exposing dentine (Watts \& Addy, 2001).

Another non-invasive approach for this patient would be vital tooth bleaching which can either be home-bleaching or in-office professional bleaching treatment. One of the drawbacks of home bleaching is that it requires a long period of exposure to bleaching material (14 days) and would cause both fluorosed and non-fluorosed areas of teeth to be subjected to the bleaching agent. There is also a common complaint of post-treatment sensitivity from home bleaching which can affect patient compliance to the treatment itself (Bussadori et al., 2004; Wright, 2002). Inoffice bleaching treatment also requires multiple visits to prevent the risk of tooth hypersensitivity which can prolong the treatment if the required shade is not achieved.

The infiltration technique itself is not new, being first described by Robinson and others back in 1976 in which they use resorcinolformaldehyde as the infiltrant agent. However, due to concern regarding the toxicity of this material, it was barred from being used on the patient. A newer approach with a safer and higher penetration coefficient of the materials widen the usage of this technique in both caries control and masking dental discolouration (Paris et al., 2007; Meyer-Lueckel \& Paris, 2008). The current infiltrant agent is a clear, unfilled composite resin with low viscosity, high surface tension and low contact angles relative to the enamel which allows the infiltrant to reach the honeycomb-like porosity in the pathological layer of enamel via capillary forces (Gugnani et al., 2012). The porosities are usually filled with water and air which have a difference in the refractive index compared to the normal enamel. As the refractive index of resininfiltrated enamel is close to that of normal enamel through the 'chameleon effect' properties of the resin, less light scattering occurs and give the infiltrated enamel the appearance that is relatively close to normal enamel.

Some concerns have been raised regarding the long term colour stability and susceptibility to staining of this technique based on in-vitro studies which need to be considered and included in treatment planning. Ulrich and colleagues found that 
surface roughness of tooth treated with resin infiltration technique was higher than that of sound enamel in addition to demineralized enamel and this is supported by recent studies (Ulrich et al., 2015; El-Meligy et al., 2021). The micro-roughness of resin infiltrated surface can act as a medium for extrinsic staining agents and plaque to accumulate which over time will result in significant alterations in colour. The discolouration issue with resin infiltration also can be contributed by a high degree of water sorption of TEGDMA resin polymer in the infiltrating agent. The correlation between high water sorption and colour stability and discolouration issues are well documented in the past (Shintani et al., 1985). Polishing the resin infiltrated surface has been suggested to improve the surface roughness in reducing the unwanted ageing process due to staining challenges (Borges et al., 2014). However, Mueller and colleagues found that it is difficult to achieve a high degree of polishability as a lack of improvement was noted even after the infiltrated surface was subjected to polishing with Sof-Lex (3M ESPE) finishing and polishing system (Mueller et al., 2011). The excess infiltrant agent also can be wiped off before polymerization to reduce the chance of decay from plaque accumulation at the residual resin layer (Paris et al., 2006). Wang and others also suggest that optimal maintenance of oral hygiene is imperative in ensuring a good aesthetic appearance after completing the resin infiltration treatment (Wang et al., 2020).

Future studies may be needed to study the correlation of severity of the lesion and time of applications on colour stability of the resin-infiltrated tooth. This may aid in establishing accurate guidance on case selection and strict protocol to follow in ensuring the success of this treatment modality. The clinical outcome of the treatment showed acceptable results by patients regarding the aesthetic appearance of teeth in addition to duration and cost of treatment. However, in the authors' view, the result can be improved with a combination approach of other treatment modalities such as tooth bleaching and microabrasion.

\section{Conclusions}

This case report demonstrated that resin infiltration is a valuable option in treating mild to moderate severity of dental fluorosis, improving the appearance of the tooth by masking white spots in one appointment. It is also can be considered as a safe and minimally invasive approach in managing the unaesthetic appearance of teeth with dental fluorosis.

\section{References}

Alshammary, F., Siddiqui, A. A., Al-Enizy, A. S., Almalaq, S. A. S., Amin, J., Rathore, H. A., Alshammary, F. A., \& Alam, M. K. (2020). Prevalence of dental fluorosis in Saudi Arabia: a meta-analysis. Pesquisa Brasileira Em Odontopediatria e Clínica Integrada, 20, 1-10.

Aoba, T., \& Fejerskov, 0. (2002). Dental fluorosis: Chemistry and biology. Critical Reviews in Oral Biology and Medicine, 13(2), 155-170.

Auschill, T. M., Schmidt, K. E., \& Arweiler, N. B. (2015). Resin infiltration for aesthetic improvement of mild to moderate fluorosis: A six-month follow-up case report. Oral Health \& Preventive Dentistry, 13(4), 317-322.

Bertassoni, L. E., Martin, J. M. H., Torno, V., Vieira, S., Rached, R. N., \& Mazur, R. F. (2008). In-office dental bleaching and enamel microabrasion for fluorosis treatment. Journal of Clinical Pediatric Dentistry, 32(3), 185-188.

Borges, A. B., Caneppele, T. M. F., Luz, M., Pucci, C. R., \& Torres, C. R. G. (2014). Colour stability of resin used for caries infiltration after exposure to different staining solutions. Operative Dentistry, 39(4), 433440.

Bussadori, S. K., Do Rego, M. A., Da Silva, P. E., Pinto, M. M., \& Pinto, A. C. G. (2004). Esthetic alternative for fluorosis blemishes with the usage of a dual bleaching system based on hydrogen peroxide at 35\%. Journal of Clinical Pediatric Dentistry, 28(2), 143-146.

Crawford, P. J. M., \& Aldred, M. J. (1992). X-linked amelogenesis imperfecta. Presentation of two kindreds and a review of the literature. Oral Surgery, Oral Medicine, Oral Pathology, 73(4), 449455.

Denbesten, Pamela, \& Li, W. (2011). Chronic fluoride toxicity: Dental fluorosis. Monographs in Oral Science, 22, 81-96.

Denbesten, PK, Crenshaw, M., \& Wilson, M. (1985). Changes in the fluoride-induced modulation of maturation stage ameloblasts of rats. Journal of Dental Research, 64(12), 1365-1370.

Duarte-Rodrigues, L., Ramos-Jorge, M. L., Alves-Duarte, A. C., Fonseca-Silva, T., Flores-Mir, C., \& Marques, L. S. (2020). Oral disorders associated with the experience of verbal bullying among Brazilian school-aged children: A case-control study. Journal 
of the American Dental Association, 151(6), 399406.

El Meligy, O. A. E. S., Alamoudi, N. M., Eldin Ibrahim, S. T., Felemban, O. M., \& Al-Tuwirqi, A. A. (2021). Effect of resin infiltration application on early proximal caries lesions in vitro. Journal of Dental Sciences, 16(1), 296-303.

Fejerskov, O., Manji, F., \& Baelum, V. (1990). The nature and mechanisms of dental fluorosis in man. Journal of Dental Research, 69(SPEC. ISS. FEB.), 692-700.

Frencken, J. E., Peters, M. C., Manton, D. J., Leal, S. C., Gordan, V. V., \& Eden, E. (2012). Minimal intervention dentistry for managing dental caries A review: Report of a FDI task group. International Dental Journal, 62 (5), 223-243.

Giambro, N. J., Prostak, K., \& Besten, P. Den. (1995). Characterization of fluorosed human enamel by color reflectance, ultrastructure, and elemental composition. Caries Research, 29(4), 251-257.

Gugnani, N., Pandit, I., Gupta, M., \& Josan, R. (2012). Caries infiltration of noncavitated white spot lesions: A novel approach for immediate esthetic improvement. Contemporary Clinical Dentistry, 3(6), 199.

Karim, F. A., Yuzadi, Z., Yusof, M., Azlida, N., \& Nor, M. (2021). Prevalence of dental fluorosis and its associated factors after cessation of water fluoridation. Malaysian Journal of Medicine and Health Sciences, 17 (1), 68-76.

Kugel, G., Arsenault, P., \& Papas, A. (2009). Treatment modalities for caries management, including a new resin infiltration system. Compendium of Continuing Education in Dentistry (Jamesburg, N.J. : 1995), 30 Spec No 3, 1-10; quiz 11.

McKay, F. (1952). The study of mottled enamel (dental fluorosis). Journal of the American Dental Association (1939), 44(2), 133-137.

Meyer-Lueckel, H., \& Paris, S. (2008). Improved resin infiltration of natural caries lesions. Journal of Dental Research, 87(12), 1112-1116.

Mueller, J., Yang, F., Neumann, K., \& Kielbassa, A. (2011). Surface tridimensional topography analysis of materials and finishing procedures after resinous infiltration of subsurface bovine enamel lesions. Quintessence International (Berlin, Germany : 1985), 42(2), 135-147.

Narayanan, R., Prabhuji, M. L. V., Paramashivaiah, R., \& Bhavikatti, S. K. (2019). Low-level laser therapy in combination with desensitising agent reduces dentin hypersensitivity in fluorotic and nonfluorotic teeth - A randomised, controlled, doubleblind clinical trial. Oral Health and Preventive Dentistry, 17(6), 547-556.

Paris, S., Meyer-Lueckel, H., \& Kielbassa, A. M. (2007). Resin infiltration of natural caries lesions. Journal of Dental Research, 86(7), 662-666.

Paris, S., Meyer-Lueckel, H., Mueller, J., Hummel, M., \& Kielbassa, A. (2006). Progression of sealed initial bovine enamel lesions under demineralizing conditions in vitro. Caries Research, 40(2), 124129.

Robinson, C., Connell, S., Kirkham, J., Brookes, S. J., Shore, R. C., \& Smith, A. M. (2004). The effect of fluoride on the developing tooth. Caries Research, 38, 268-276.
Shenoi, P., Kandhari, A., \& Gunwal, M. (2012). Esthetic Enhancement of Discolored Teeth by Macroabrasion Microabrasion and its psychological impact on patients-A case series. Indian Journal of Multidisciplinary Dentistry, 2(1), 388-392.

Sherwood, I. A. (2010). Fluorosis varied treatment options. Journal of Conservative Dentistry, 13(1), 47-53.

Shintani, H., Satou, J., Satou, N., Hayashihara, H., \& Inoue, T. (1985). Effects of various finishing methods on staining and accumulation of Streptococcus mutans HS-6 on composite resins. Dental Materials: Official Publication of the Academy of Dental Materials, 1(6), 225-227.

Tan, B. S., Razak, I. A., \& Foo, L. C. (2005). Fluorosis prevalence among schoolchildren in a fluoridated community in Malaysia. Community Dental Health, 22(1), 35-39.

Tirlet, G., Chabouis, H. F., \& Attal, J. P. (2013). Infiltration, a new therapy for masking enamel white spots: a 19-month follow-up case series. The European Journal of Esthetic Dentistry: Official Journal of the European Academy of Esthetic Dentistry, 8(2), 180-190.

Ulrich, I., Mueller, J., Wolgin, M., Frank, W., \& Kielbassa, A. M. (2015). Tridimensional surface roughness analysis after resin infiltration of (deproteinized) natural subsurface carious lesions. Clinical Oral Investigations, 19(6), 1473-1483.

Wang, Q., Meng, Q., \& Meng, J. (2020). Minimally invasive esthetic management of dental fluorosis: a case report. Journal of International Medical Research, 48(10), 300060520967538.

Watts, A., \& Addy, M. (2001). Tooth discolouration and staining: A review of the literature. British Dental Journal, 190(6), 309-316.

Wiegand, A., Stawarczyk, B., Kolakovic, M., Hämmerle, C. H. F., Attin, T., \& Schmidlin, P. R. (2011). Adhesive performance of a caries infiltrant on sound and demineralised enamel. Journal of Dentistry, 39(2), 117-121.

Wright, J. T. (2002). The etch-bleach-seal technique for managing stained enamel defects in young permanent incisors. Pediatric Dentistry, 24(3), 249-252. 